\title{
Screening for Alcohol Use and Brief Counseling of Adults - 13 States and the District of Columbia, 2017
}

\author{
Lela R. McKnight-Eily, $\mathrm{PhD}^{1}$; Catherine A. Okoro, $\mathrm{PhD}^{2}$; Khadija Turay, $\mathrm{PhD}^{3}$; Cristian Acero, $\mathrm{MPH}^{1}$; Dan Hungerford, DrPH ${ }^{1}$
}

Binge drinking* is a leading preventable public health problem. From 2006 to 2010, binge drinking contributed to approximately 49,000 annual deaths resulting from acute conditions (e.g., injuries and violence) (1). Binge drinking also increases the risk for adverse health conditions, including some chronic diseases (e.g., breast cancer) and fetal alcohol spectrum disorders (2). In 2004, 2013, and again in 2018, for all U.S. adults aged $\geq 18$ years in primary care, the U.S. Preventive Services Task Force (USPSTF) recommended alcohol screening and brief intervention (alcohol SBI) or counseling for persons whose screening indicated drinking in excess of recommended limits or in ways that increase risk for poor health outcomes (3-5). However, previous CDC surveillance data indicate that patients report rarely talking to their provider about alcohol use, ${ }^{\dagger}$ and alcohol SBI is traditionally delivered through conversation. CDC recently analyzed 2017 data from the Behavioral Risk Factor Surveillance System (BRFSS) survey's five-question module, which asked adults in 13 states $^{\S}$ and the District of Columbia (DC) about the delivery of alcohol SBI during their most recent checkup in the past 2 years. Overall, $81.4 \%$ of adults (age-standardized estimate) reported being asked about alcohol use by a health professional in person or on a form during a checkup in the past 2 years, but only $37.8 \%$ reported being asked a question about binge-level alcohol consumption, which is included on USPSTF recommended instruments (3). Among module respondents who were asked about alcohol use at a checkup in the past 2 years and reported current binge drinking (past 30 days) at time of survey, only $41.7 \%$ were advised about the harms of drinking too much at a checkup in the past 2 years, and only $20.1 \%$ were advised to reduce or quit drinking at a checkup in the past 2 years. These findings suggest that missed opportunities remain for health care providers to intervene with patients who report binge drinking. Working to implement alcohol SBI at a systems level, including the provision of the new Healthcare Effectiveness Data Information Set (HEDIS)

\footnotetext{
* The National Institute on Alcohol Abuse and Alcoholism defines binge drinking as a pattern of drinking that brings blood alcohol concentration levels to $0.08 \mathrm{~g} / \mathrm{dL}$. This typically occurs after four drinks for women and five drinks for men, in approximately 2 hours. https://pubs.niaaa.nih.gov/publications/ Newsletter/winter2004/Newsletter_Number3.pdf.

${ }^{\dagger}$ https://www.cdc.gov/vitalsigns/alcohol-screening-counseling/index.html.

$\$$ Alabama, Alaska, Arizona, Arkansas, California, Colorado, Connecticut, Kansas, Nebraska, Nevada, New Hampshire, Tennessee, and Wisconsin.
}

measure, Unhealthy Alcohol Use Screening and Follow-Up, can improve alcohol SBI's use and benefit in primary care.

BRFSS is an ongoing state-based, random-digit-dialed telephone survey of the noninstitutionalized U.S. adult population aged $\geq 18$ years in all 50 states, DC, and participating U.S. territories. Information is collected on various health conditions, health practices, and risk behaviors, including alcohol use. CDC analyzed 2017 data from the 13 states and DC that administered an optional alcohol SBI module. All BRFSS respondents are asked about the timing of their last routine checkup. Those who had a checkup in the past 2 years were asked alcohol SBI module questions. 9 All module respondents were asked four questions: 1) "You told me earlier that your last routine checkup was [within the past year/within the past 2 years]. At that checkup, were you asked in person or on a form if you drink alcohol?"; 2) "Did the health care provider ask you in person or on a form how much you drink?"; 3) "Did the health care provider specifically ask whether you drank ( 5 for men/4 for women) or more alcoholic drinks on an occasion?"; and 4) "Were you offered advice about what level of drinking is harmful or risky for your health?" Persons who responded affirmatively to any of the first three questions (alcohol use screening-related) were also asked "Healthcare providers may also advise patients to drink less for various reasons. At your last routine checkup, were you advised to reduce or quit your drinking?" to assess brief counseling. BRFSS assesses current binge drinking by report of drinking four (women) or five (men) or more drinks on one or more occasions during the past 30 days.

Analyses were conducted to account for BRFSS's complex sampling design. Weighted crude and age-standardized prevalence estimates of responses to alcohol SBI module questions were calculated. Estimates were stratified by demographic characteristics and selected drinking patterns. Subanalyses were performed among alcohol SBI module respondents who indicated that they had been asked at least one of three alcohol use screening-related questions in the past 2 years and who reported current binge drinking (in the past 30 days) at time of survey. Only age-standardized estimates are included in the text of this report. Wald chi-squared tests were used to determine

\footnotetext{
The module lead-in question was "Healthcare providers may ask during routine checkups about behaviors like alcohol use, whether you drink or not. We want to know about their questions."
} 
significant within-group differences. SUDAAN (version 11.0.3; RTI International) was used for analyses. Only significant differences are reported. The median cooperation rate** for the 14 sites was $71.7 \%$, and median response rate was $43.8 \%$.

Overall, $81.4 \%$ of module respondents indicated being asked by their health care provider about alcohol use in person or by form, $71.8 \%$ reported being asked how much they drink, and 37.8\% reported being asked about binge drinking (Table 1). The prevalence of module respondents being asked about binge drinking was higher among males (40.1\%) and persons with less than a high school diploma (46.2\%) than among females $(36.0 \%)$ and persons with higher levels of education $(36.1 \%$ [college or technical school] to $37.1 \%$ [high school diploma]). A higher percentage of persons with a household income $<200 \%$ of the federal poverty level were asked about binge drinking than were persons with an income $\geq 200 \%$ of the federal poverty level. Hispanic adults reported being asked about binge drinking more than other racial/ethnic groups. Prevalence of being asked about binge drinking was also higher among module respondents who reported binge drinking (47.3\%) than among those who did not (36.1\%).

Among module respondents who were asked at least one of the alcohol use screening-related questions at a checkup in the past 2 years and reported current binge drinking (past 30 days) at time of survey, only $41.7 \%$ were advised about the harms of drinking too much at a checkup in the past 2 years, and only $20.1 \%$ were advised to reduce or quit drinking at a checkup in the past 2 years (Table 2). Among module respondents who were asked at least one of the alcohol use screening-related questions and reported current binge drinking in the past 30 days at time of survey, the prevalence of being advised to reduce drinking at a checkup in the past 2 years was higher among males (24.1\%), persons with a disability $(28.2 \%)$, persons with less than a high school education $(38.2 \%)$, persons with income $<100 \%$ of the federal poverty level (37.5\%), and those without health insurance coverage (36.2\%) than among their counterparts. Prevalence was also higher among Hispanic adults $(28.5 \%)$ than among white adults $(16.8 \%)$.

\section{Discussion}

In 2017 , although $81 \%$ of U.S. adults reported being asked by their health care provider about alcohol use, only $38 \%$ reported being asked about binge drinking during a checkup in the past 2 years, based on BRFSS data from 13 states and DC. Fewer than half (42\%) of module respondents who were asked about alcohol use at a checkup in the past 2 years and reported current binge drinking (past 30 days) at time of survey were

\footnotetext{
** The cooperation rate is the number of complete and partial complete interviews divided by the number of contacted and eligible respondents.
}

advised of harmful drinking levels; almost $80 \%$ (four of five persons) received no advice to reduce their drinking (only 20\% were advised to reduce their drinking). Previous overall 2014 estimates, using the BRFSS alcohol SBI module from 17 states and DC (6), were similar to overall 2017 findings, but not directly comparable because of differences in states repeating the module in 2014 and 2017 (only five states implemented the module both years). State-level trend analysis might occur in future reports. An assessment of binge-level consumption is included on USPSTF-recommended screening tools (5).

Screening alone is not effective at reducing binge drinking (5). Brief counseling involves feedback based on screening results, a conversation about the dangers of excessive drinking on the patient's health, and development of a plan to reduce drinking if the patient chooses to do so $(5,7)$. Behavioral counseling is a necessary component of alcohol SBI for reduction in consumption and adherence to drinking limits (5). Persons with dependence are to be referred to treatment, but referral might not occur, or patients might not accept the referral, obtain treatment, or respond to treatment $(5,7)$. The Substance Abuse and Mental Health Services Administration, which has long promoted SBI through grant programs, has a treatment locator $^{\dagger \dagger}$ to assist with the referral process. A 2018 USPSTF review found that "Among adults identified through screening, counseling interventions to reduce unhealthy alcohol use were associated with reductions in alcohol use (by a mean of 1.6 drinks/wk) and in the odds of exceeding recommended drinking limits (by 40\%) and heavy use episodes (by 33\%) at 6 to 12 months of follow-up....Among pregnant women, counseling interventions were associated with an odds ratio of 2.26 for remaining abstinent from alcohol during pregnancy." (5).

The demographic differences in this report might be a consequence of some adults having more contact with health systems, such as those with a disability (8) or veterans, which could increase their likelihood of receipt of alcohol SBI. In addition, screening practices might vary in health care systems that have systematically implemented alcohol SBI (e.g., U.S. Department of Veterans Affairs and federally qualified health centers) (9).

Health system changes, such as the acceptance of a new 2018 HEDIS measure: Unhealthy Alcohol Use Screening and Follow-Up, ${ }^{\$ S}$ might increase the provision of alcohol SBI.

\footnotetext{
†† https://findtreatment.samhsa.gov/.

$\$ \$$ The 2018 Healthcare Effectiveness Data Information Set (HEDIS) measure, Unhealthy Alcohol Use Screening and Follow-Up, was approved as a first-year measure in June 2017 by the National Committee for Quality Assurance as a 2018 HEDIS measure. The testing and submission of this measure into HEDIS was supported by the Substance Abuse and Mental Health Services Administration. The measure uses standardized tools for alcohol SBI and for those who screen positive, the percentage who receive brief counseling or other follow-up within 2 months of the positive screen is documented. https://www.ncqa.org/hedis/reports-and-research/ hedis-measure-unhealthy-alcohol-use-screening-and-follow-up/.
} 
TABLE 1. Weighted crude and age-standardized* percentages of U.S. adults who reported being asked an alcohol use screening-related question by a health care provider at last routine checkup in the past 2 years - Behavioral Risk Factor Surveillance System, 13 states ${ }^{\dagger}$ and the District of Columbia, 2017

\begin{tabular}{|c|c|c|c|c|c|c|c|c|c|}
\hline \multirow[b]{2}{*}{ Characteristic } & \multicolumn{3}{|c|}{$\begin{array}{l}\text { Asked about alcohol use } \\
\text { (affirmative to question 1) }\end{array}$} & \multicolumn{3}{|c|}{$\begin{array}{l}\text { Asked how much alcohol } \\
\text { (affirmative to question 2) }\end{array}$} & \multicolumn{3}{|c|}{$\begin{array}{l}\text { Asked about binge drinking } \\
\text { (affirmative to question } 3 \text { ) }\end{array}$} \\
\hline & $\begin{array}{l}\text { Sample } \\
\text { size }\end{array}$ & $\begin{array}{c}\text { Crude } \\
\%(95 \% \mathrm{Cl})\end{array}$ & $\begin{array}{c}\text { Age-standardized } \\
\%(95 \% \mathrm{Cl})\end{array}$ & Sample size & $\begin{array}{c}\text { Crude } \\
\%(95 \% \mathrm{Cl})\end{array}$ & $\begin{array}{c}\text { Age-standardized } \\
\%(95 \% \mathrm{Cl})\end{array}$ & Sample size & $\begin{array}{c}\text { Crude } \\
\%(95 \% \mathrm{Cl})\end{array}$ & $\begin{array}{c}\text { Age-standardized } \\
\%(95 \% \mathrm{Cl})\end{array}$ \\
\hline Total & 65,887 & $79.7(79.0-80.5)$ & $81.4(80.7-82.1)$ & 65,913 & $70.0(69.2-70.8)$ & 71.8 (70.9-72.6) & 59,215 & $35.7(34.8-36.6)$ & $37.8(36.9-38.8)$ \\
\hline \multicolumn{10}{|l|}{ Sex } \\
\hline Male & 27,725 & $79.7(78.6-80.8)$ & $81.0(79.9-82.1)$ & 27,663 & $70.1(68.9-71.3)$ & $71.5(70.3-72.7)$ & 24,942 & $37.9(36.6-39.3)$ & $40.1(38.6-41.5)$ \\
\hline Female & 38,114 & $79.7(78.7-80.7)$ & $81.8(80.9-82.7)$ & 38,200 & $69.9(68.8-71.0)$ & $72.2(71.0-73.3)$ & 34,223 & $33.8(32.5-35.0)$ & $36.0(34.6-37.3)$ \\
\hline \multicolumn{10}{|l|}{ Age group (yrs) } \\
\hline $18-24$ & 3,018 & $82.3(79.7-84.7)$ & - & 2,953 & $65.5(62.3-68.5)$ & - & 2,750 & $30.6(27.7-33.6)$ & - \\
\hline $25-34$ & 5,122 & $88.7(86.9-90.3)$ & - & 5,032 & $80.9(78.7-82.9)$ & - & 4,385 & $48.9(46.2-51.7)$ & - \\
\hline $35-44$ & 6,867 & $86.7(85.1-88.2)$ & - & 6,785 & $79.2(77.1-81.1)$ & - & 5,713 & $45.7(43.2-48.3)$ & - \\
\hline $45-64$ & 25,175 & $80.6(79.4-81.8)$ & - & 24,982 & $71.8(70.4-73.1)$ & - & 21,977 & $35.9(34.4-37.3)$ & - \\
\hline$\geq 65$ & 25,705 & $67.3(65.7-68.9)$ & - & 26,161 & $57.4(55.8-58.9)$ & - & 24,390 & $24.8(23.4-26.3)$ & - \\
\hline \multicolumn{10}{|l|}{ Race/Ethnicity ${ }^{\S}$} \\
\hline White & 49,451 & $79.9(79.1-80.6)$ & $83.2(82.5-84.0)$ & 49,605 & $71.6(70.8-72.4)$ & $75.2(74.3-76.1)$ & 43,948 & $31.6(30.6-32.5)$ & $35.7(34.5-36.9)$ \\
\hline Black & 5,993 & $76.2(73.3-78.8)$ & $77.2(74.7-79.6)$ & 5,967 & $64.9(61.8-67.8)$ & $65.7(62.9-68.4)$ & 5,632 & $36.5(33.7-39.5)$ & $36.8(34.0-39.7)$ \\
\hline Hispanic & 5,381 & $83.8(82.1-85.3)$ & $82.4(80.7-84.0)$ & 5,307 & $70.7(68.6-72.7)$ & $69.7(67.6-71.8)$ & 4,985 & $46.6(44.2-49.0)$ & $46.9(44.6-49.2)$ \\
\hline $\mathrm{A} / \mathrm{PI}$ & 1,158 & $71.8(66.4-76.5)$ & $71.7(66.3-76.6)$ & 1,150 & $61.4(55.9-66.5)$ & $61.9(56.5-67.1)$ & 1,084 & $31.8(27.0-37.1)$ & $32.1(27.3-37.3)$ \\
\hline $\mathrm{Al} / \mathrm{AN}$ & 1,271 & $82.0(77.6-85.6)$ & $82.8(78.6-86.3)$ & 1,264 & $71.7(66.4-76.4)$ & 73.6 (68.9-77.9) & 1,203 & $42.6(36.8-48.6)$ & $45.1(39.8-50.5)$ \\
\hline $\begin{array}{l}\text { Other race/ } \\
\text { Multiracial }\end{array}$ & 1,586 & $81.7(77.7-85.2)$ & $82.5(78.5-85.9)$ & 1,573 & $74.0(69.1-78.4)$ & $75.2(70.8-79.2)$ & 1,417 & $37.4(32.0-43.1)$ & $38.4(33.1-43.9)$ \\
\hline \multicolumn{10}{|l|}{ Education level } \\
\hline $\begin{array}{l}\text { Less than high } \\
\text { school diploma }\end{array}$ & 4,315 & $77.2(74.9-79.3)$ & $78.1(75.6-80.5)$ & 4,279 & $62.5(59.7-65.1)$ & $63.6(60.6-66.6)$ & 4,163 & $45.4(42.5-48.3)$ & $46.2(43.2-49.3)$ \\
\hline $\begin{array}{l}\text { High school } \\
\text { diploma }\end{array}$ & 16,753 & $76.9(75.4-78.3)$ & $78.9(77.4-80.2)$ & 16,824 & $64.5(62.8-66.1)$ & $67.3(65.6-68.9)$ & 15,766 & $34.0(32.4-35.7)$ & $37.1(35.3-39.0)$ \\
\hline $\begin{array}{l}\text { College or } \\
\text { technical school }\end{array}$ & 44,641 & $81.5(80.6-82.4)$ & $83.2(82.4-84.1)$ & 44,630 & $74.1(73.1-75.1)$ & $75.8(74.8-76.8)$ & 39,121 & $34.0(32.9-35.1)$ & $36.1(34.9-37.3)$ \\
\hline \multicolumn{10}{|c|}{ Federal poverty level, \%ף } \\
\hline$<100$ & 5,896 & $79.0(76.9-81.0)$ & $78.1(75.9-80.1)$ & 5,850 & $66.6(64.1-69.0)$ & $65.9(63.4-68.3)$ & 5,570 & $44.0(41.3-46.7)$ & $44.0(41.4-46.6)$ \\
\hline $100-199$ & 12,773 & $77.5(75.8-79.1)$ & $80.2(78.5-81.8)$ & 12,842 & $66.6(64.7-68.5)$ & $69.7(67.7-71.6)$ & 12,049 & $37.8(35.8-39.9)$ & $41.1(38.8-43.5)$ \\
\hline$\geq 200$ & 36,949 & $82.3(81.3-83.2)$ & $83.9(82.9-84.9)$ & 36,873 & $74.5(73.4-75.6)$ & $75.6(74.4-76.7)$ & 32,219 & $33.7(32.5-34.9)$ & $36.0(34.6-37.4)$ \\
\hline Unknown & 10,269 & $74.2(72.1-76.3)$ & $76.6(74.3-78.7)$ & 10,348 & $61.8(59.4-64.1)$ & $65.2(62.5-67.8)$ & 9,377 & $31.4(29.2-33.8)$ & $35.8(33.0-38.8)$ \\
\hline \multicolumn{10}{|l|}{ Veteran } \\
\hline Yes & 9,017 & $79.4(77.3-81.4)$ & $86.6(84.2-88.7)$ & 9,071 & $71.2(68.8-73.5)$ & $78.8(75.0-82.1)$ & 8,277 & $37.2(35.0-39.6)$ & $48.5(44.4-52.7)$ \\
\hline No & 56,801 & $79.8(79.0-80.5)$ & $80.9(80.2-81.7)$ & 56,772 & $69.9(69.0-70.7)$ & $71.2(70.3-72.1)$ & 50,876 & $35.5(34.5-36.5)$ & $37.1(36.1-38.2)$ \\
\hline \multicolumn{10}{|l|}{ Disability status*** } \\
\hline Yes & 20,225 & $75.0(73.7-76.3)$ & $79.4(78.0-80.8)$ & 20,356 & $64.6(63.1-66.0)$ & $69.4(67.6-71.1)$ & 19,149 & $34.3(32.8-35.8)$ & $38.8(36.9-40.8)$ \\
\hline No & 45,143 & $81.6(80.7-82.4)$ & $82.2(81.4-83.1)$ & 45,055 & $72.2(71.2-73.2)$ & $72.9(71.9-73.9)$ & 39,614 & $36.4(35.3-37.5)$ & $37.6(36.4-38.7)$ \\
\hline \multicolumn{10}{|c|}{ Health insurance coverage } \\
\hline Yes & 62,282 & $79.8(79.0-80.6)$ & $81.9(81.2-82.7)$ & 62,369 & $70.5(69.6-71.3)$ & $72.8(71.9-73.7)$ & 55,905 & $34.9(34.0-35.8)$ & $37.4(36.3-38.4)$ \\
\hline No & 3,416 & $78.9(76.2-81.3)$ & $76.9(74.1-79.5)$ & 3,359 & $65.7(62.6-68.6)$ & $62.8(59.6-65.8)$ & 3,141 & $45.8(42.5-49.1)$ & $44.5(41.2-47.8)$ \\
\hline \multicolumn{10}{|c|}{ Reported current drinking } \\
\hline Yes & 35,154 & $85.0(84.1-85.8)$ & $85.8(84.9-86.6)$ & 34,990 & $78.6(77.5-79.6)$ & $79.2(78.2-80.2)$ & 30,432 & $39.4(38.1-40.7)$ & $40.7(39.3-42.1)$ \\
\hline No & 30,076 & $73.5(72.3-74.6)$ & $76.4(75.2-77.6)$ & 30,271 & $59.8(58.5-61.1)$ & $63.1(61.7-64.5)$ & 28,198 & $31.5(30.3-32.8)$ & $34.7(33.3-36.2)$ \\
\hline \multicolumn{10}{|c|}{ Reported binge drinking ${ }^{\dagger \dagger}$} \\
\hline Yes & 7,807 & $89.3(87.9-90.6)$ & $88.7(87.2-90.0)$ & 7,725 & $83.8(82.1-85.4)$ & $83.6(81.8-85.2)$ & 6,741 & $47.9(45.2-50.5)$ & $47.3(44.7-50.0)$ \\
\hline No & 57,042 & $78.0(77.2-78.9)$ & $80.2(79.4-81.0)$ & 57,162 & $67.6(66.7-68.5)$ & $69.8(68.8-70.8)$ & 51,553 & $33.5(32.6-34.5)$ & $36.1(35.0-37.2)$ \\
\hline
\end{tabular}

Abbreviations: AI/AN = American Indian/Alaska Native; A/PI = Asian/Pacific Islander; $\mathrm{Cl}=$ confidence interval; $F \mathrm{PL}=$ federal poverty level.

* Estimates are age-standardized to the 2000 projected population for the United States.

† Alabama, Alaska, Arizona, Arkansas, California, Colorado, Connecticut, Kansas, Nebraska, Nevada, New Hampshire, Tennessee, and Wisconsin.

$\S$ Persons in all racial groups were non-Hispanic. Persons who self-identified as Hispanic might have been of any race.

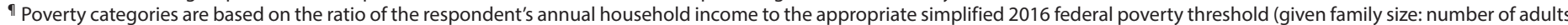

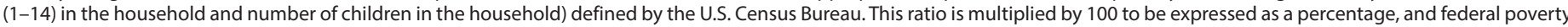

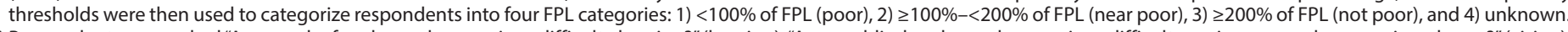

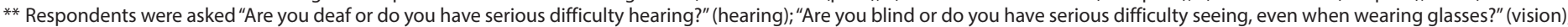

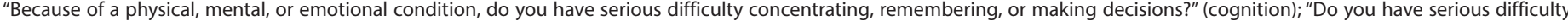

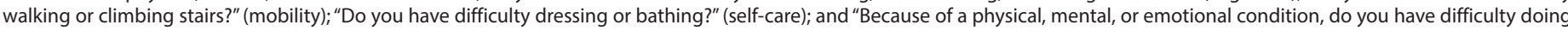

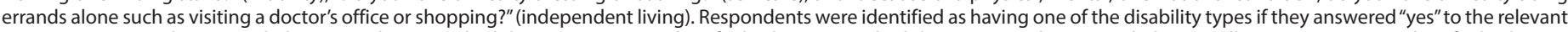

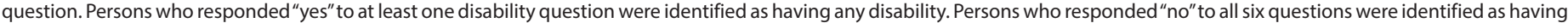
no disability. Missing responses and respondents who answered "don't know" or who declined to answer were excluded.

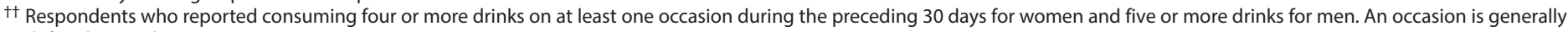
defined as $2-3$ hours. 
TABLE 2. Weighted crude and age-standardized* estimates of being advised about harmful or risky drinking levels and to reduce the level of drinking, among U.S. adults who reported being asked an alcohol use screening-related question by a health care provider at last routine checkup in the past 2 years and reported current binge drinking in the past 30 days at time of survey ${ }^{\dagger}$ - Behavioral Risk Factor Surveillance System, 13 states $^{\S}$ and the District of Columbia, 2017

\begin{tabular}{|c|c|c|c|c|c|c|}
\hline \multirow[b]{3}{*}{ Characteristic } & \multicolumn{6}{|c|}{$\begin{array}{l}\text { Adults who were asked an alcohol use screening-related question" and reported current binge drinking in the past } \\
\qquad 30 \text { days at time of survey }\end{array}$} \\
\hline & \multicolumn{3}{|c|}{ Advised about level of drinking harmful/risky to health" } & \multicolumn{3}{|c|}{ Advised to reduce drinking } \\
\hline & Sample size & $\begin{array}{l}\text { Crude } \\
\%(95 \% \mathrm{Cl})\end{array}$ & $\begin{array}{l}\text { Age-standardized } \\
\quad \%(95 \% \mathrm{Cl})\end{array}$ & $\begin{array}{l}\text { Sample } \\
\text { size }\end{array}$ & $\begin{array}{l}\text { Crude } \\
\%(95 \% \mathrm{Cl})\end{array}$ & $\begin{array}{l}\text { Age-standardized } \\
\quad \%(95 \% \mathrm{Cl})\end{array}$ \\
\hline Total & 6,811 & $41.8(39.1-44.4)$ & $41.7(39.0-44.4)$ & 6,943 & $20.6(18.6-22.6)$ & $20.1(18.2-22.2)$ \\
\hline $\begin{array}{l}\text { Sex } \\
\text { Male } \\
\text { Female }\end{array}$ & $\begin{array}{l}3,924 \\
2,883\end{array}$ & $\begin{array}{l}47.4(44.0-50.8) \\
32.7(28.9-36.7)\end{array}$ & $\begin{array}{l}46.7(43.2-50.3) \\
33.8(29.9-37.9)\end{array}$ & $\begin{array}{l}4,004 \\
2,935\end{array}$ & $\begin{array}{l}24.9(22.3-27.8) \\
13.6(11.0-16.5)\end{array}$ & $\begin{array}{l}24.1(21.5-26.8) \\
13.7(10.9-17.1)\end{array}$ \\
\hline $\begin{array}{l}\text { Age group (yrs) } \\
18-24 \\
25-34 \\
35-44 \\
45-64 \\
\geq 65\end{array}$ & $\begin{array}{r}655 \\
1,187 \\
1,186 \\
2,754 \\
1,029\end{array}$ & $\begin{array}{l}43.2(35.6-51.2) \\
42.4(37.1-47.8) \\
37.6(31.5-44.0) \\
42.7(38.8-46.7) \\
43.3(35.2-51.7)\end{array}$ & $\begin{array}{l}- \\
- \\
- \\
-\end{array}$ & $\begin{array}{r}665 \\
1,214 \\
1,213 \\
2,812 \\
1,039\end{array}$ & $\begin{array}{l}16.6(12.2-22.2) \\
20.8(16.8-25.4) \\
17.1(13.5-21.4) \\
25.9(22.4-29.8) \\
15.7(10.9-22.3)\end{array}$ & $\begin{array}{l}- \\
- \\
- \\
-\end{array}$ \\
\hline $\begin{array}{l}\text { Race/Ethnicity** } \\
\text { White } \\
\text { Black } \\
\text { Hispanic } \\
\text { A/PI } \\
\text { Al/AN } \\
\text { Other race/Multiracial }\end{array}$ & $\begin{array}{r}5,046 \\
511 \\
720 \\
105 \\
161 \\
189\end{array}$ & $\begin{array}{l}40.9(38.1-43.8) \\
43.0(34.2-52.3) \\
42.6(36.3-49.1) \\
45.9(29.3-63.4) \\
58.7(41.6-74.0) \\
35.6(24.2-49.0)\end{array}$ & $\begin{array}{l}40.9(38.0-43.9) \\
44.8(35.7-54.4) \\
42.6(35.2-50.4) \\
50.5(33.9-67.1) \\
57.8(44.8-69.7) \\
34.9(24.4-47.1)\end{array}$ & $\begin{array}{r}5,161 \\
514 \\
729 \\
104 \\
163 \\
190\end{array}$ & $\begin{array}{r}16.8(15.0-18.9) \\
20.1(14.5-27.1) \\
28.8(23.4-34.8) \\
19.9(10.7-33.9)^{\dagger+} \\
\mathrm{N} / \mathrm{A}^{\S \S} \\
23.6(13.8-37.4)^{\dagger+}\end{array}$ & $\begin{array}{r}16.8(14.9-19.0) \\
19.9(15.0-25.9) \\
28.5(23.4-34.2) \\
\mathrm{N} / \mathrm{A}^{\S \S} \\
27.9(17.2-41.9)^{\dagger \dagger} \\
24.6(15.1-37.5)^{\dagger \dagger}\end{array}$ \\
\hline $\begin{array}{l}\text { Education level } \\
\text { Less than high school diploma } \\
\text { High school diploma } \\
\text { College or technical school }\end{array}$ & $\begin{array}{r}340 \\
1,659 \\
4,806\end{array}$ & $\begin{array}{l}61.9(52.5-70.4) \\
40.4(35.1-45.8) \\
38.5(35.7-41.4)\end{array}$ & $\begin{array}{l}63.8(54.4-72.3) \\
40.5(35.5-45.8) \\
38.4(35.5-41.4)\end{array}$ & $\begin{array}{r}343 \\
1,682 \\
4,912\end{array}$ & $\begin{array}{l}42.4(33.2-52.2) \\
22.0(18.1-26.4) \\
16.0(14.0-18.2)\end{array}$ & $\begin{array}{l}38.2(30.0-47.1) \\
21.1(17.6-25.2) \\
16.0(13.9-18.3)\end{array}$ \\
\hline $\begin{array}{l}\text { Federal poverty level, \% } \\
<100 \\
100-199 \\
\geq 200 \\
\text { Unknown }\end{array}$ & $\begin{array}{r}578 \\
1,076 \\
4,532 \\
625\end{array}$ & $\begin{array}{l}44.9(37.3-52.8) \\
47.9(41.0-54.9) \\
39.0(36.0-42.2) \\
42.4(33.6-51.7)\end{array}$ & $\begin{array}{l}41.9(34.9-49.3) \\
47.9(41.1-54.7) \\
39.4(36.3-42.7) \\
39.8(33.3-46.8)\end{array}$ & $\begin{array}{r}583 \\
1,088 \\
4,632 \\
640\end{array}$ & $\begin{array}{l}36.2(28.9-44.1) \\
21.8(17.2-27.2) \\
16.5(14.5-18.8) \\
21.7(15.5-29.4)\end{array}$ & $\begin{array}{l}37.5(30.8-44.8) \\
21.5(17.6-26.1) \\
16.3(14.2-18.8) \\
23.6(17.6-30.9)\end{array}$ \\
\hline $\begin{array}{l}\text { Veteran } \\
\text { Yes } \\
\text { No }\end{array}$ & $\begin{array}{r}856 \\
5,951\end{array}$ & $\begin{array}{l}52.1(45.1-59.1) \\
40.6(37.8-43.4)\end{array}$ & $\begin{array}{l}53.5(45.8-61.1) \\
40.8(37.8-43.9)\end{array}$ & $\begin{array}{r}873 \\
6,066\end{array}$ & $\begin{array}{l}21.3(16.3-27.4) \\
20.5(18.4-22.7)\end{array}$ & $\begin{array}{l}21.5(16.1-28.0) \\
19.8(17.7-22.1)\end{array}$ \\
\hline $\begin{array}{l}\text { Disability status*** } \\
\text { Yes } \\
\text { No }\end{array}$ & $\begin{array}{l}1,451 \\
5,330\end{array}$ & $\begin{array}{l}50.1(44.3-56.0) \\
39.5(36.6-42.5)\end{array}$ & $\begin{array}{l}49.2(43.7-54.7) \\
39.3(36.3-42.3)\end{array}$ & $\begin{array}{l}1,472 \\
5,440\end{array}$ & $\begin{array}{l}28.7(24.1-33.9) \\
18.4(16.3-20.7)\end{array}$ & $\begin{array}{l}28.2(23.6-33.3) \\
17.8(15.8-19.9)\end{array}$ \\
\hline $\begin{array}{l}\text { Health insurance coverage } \\
\text { Yes } \\
\text { No }\end{array}$ & $\begin{array}{r}6,287 \\
507\end{array}$ & $\begin{array}{l}41.6(38.8-44.4) \\
44.3(36.9-52.1)\end{array}$ & $\begin{array}{l}41.4(38.6-44.3) \\
48.6(40.7-56.6)\end{array}$ & $\begin{array}{r}6,407 \\
518\end{array}$ & $\begin{array}{l}19.3(17.3-21.4) \\
33.1(26.1-41.0)\end{array}$ & $\begin{array}{l}18.9(16.9-21.0) \\
36.2(28.2-45.1)\end{array}$ \\
\hline
\end{tabular}

Abbreviations: Al/AN = American Indian/Alaska Native; $\mathrm{A} / \mathrm{PI}=$ Asian/Pacific Islander; $\mathrm{Cl}=$ confidence interval; $\mathrm{FPL}=$ federal poverty level; $\mathrm{N} / \mathrm{A}=$ not available.

* Estimates are age-standardized to the 2000 projected population for the United States.

${ }^{\dagger}$ Respondents who reported consuming four or more drinks on at least one occasion during the preceding 30 days for women and five or more drinks for men. An occasion is generally defined as $2-3$ hours.

$\S$ Alabama, Alaska, Arizona, Arkansas, California, Colorado, Connecticut, Kansas, Nebraska, Nevada, New Hampshire, Tennessee, and Wisconsin.

" At a checkup in the past 2 years.

** Persons in all racial groups were non-Hispanic; persons who self-identified as Hispanic might have been of any race.

†† Relative standard error $=0.20-0.30$.

$\S \S$ Estimate not available because relative standard error $>0.30$.

१ी Poverty categories are based on the ratio of the respondent's annual household income to the appropriate simplified 2016 federal poverty threshold (given family size: number of adults (1-14) in the household and number of children $(\geq 0)$ in the household) defined by the U.S. Census Bureau. This ratio is multiplied by 100 to be expressed as a percentage, and federal poverty thresholds were then used to categorize respondents into four FPL categories: 1 ) $<100 \%$ of FPL (poor), 2) $\geq 100 \%$ to $<200 \%$ of FPL (near poor), 3) $\geq 200 \%$ of FPL (not poor), and 4) unknown.

*** Respondents were asked "Are you deaf or do you have serious difficulty hearing?" (hearing); "Are you blind or do you have serious difficulty seeing, even when wearing glasses?" (vision); "Because of a physical, mental, or emotional condition, do you have serious difficulty concentrating, remembering, or making decisions?" (cognition);"Do you have serious difficulty walking or climbing stairs?" (mobility);"Do you have difficulty dressing or bathing?" (self-care); and "Because of a physical, mental, or emotional condition, do you have difficulty doing errands alone such as visiting a doctor's office or shopping?" (independent living). Respondents were identified as having one of the disability types if they answered "yes" to the relevant question. Persons who responded "yes" to at least one disability question were identified as having any disability. Persons who responded "no" to all six questions were identified as having no disability. Missing responses and respondents who answered "don't know" or who declined to answer were excluded. 
Further, federal agencies have promoted broad implementation of alcohol SBI, including CDC's funding initiatives to organizations working on fetal alcohol spectrum disorder, 99 the development of training and implementation resources, ${ }^{* * *}$ and cross-agency, medical, and private sector partnerships.

The 2015-2020 Dietary Guidelines for Americans recommends that if alcohol is consumed, it should be in moderation (up to one drink a day for women, two for men) and only by adults of legal drinking age. ${ }^{\dagger \dagger}$ The 2015-2020 Dietary Guidelines for Americans and the National Institute for Alcohol Abuse and Alcoholism also indicate or advise that some persons should not drink alcohol at all, including pregnant women $(10)$ or those who might be pregnant or persons who have certain medical conditions or are taking medications that can interact with alcohol (10).

The findings in this report are subject to at least four limitations. First, BRFSS data are self-reported, which can result in recall and social desirability biases around the period of recall for the checkup. Second, data in this report were from 14 sites, and thus, these results cannot be used to estimate the prevalence of alcohol SBI across all states and territories. Third, although respondents indicated current binge drinking in response to a BRFSS survey question, whether they reported binge drinking to their health care provider at time of checkup in the past 2 years is unknown; many respondents reported not being asked about binge drinking at a checkup in the past 2 years. Finally, the survey median response rate was only $43.8 \%$, which increases the possibility of response bias.

Binge drinking among U.S. adults continues to be a leading preventable cause of considerable morbidity and mortality (1). Alcohol SBI is an effective clinical preventive service for reducing excess alcohol use, $\$ \$ \$$ including binge consumption $(3,5,7)$. This report suggests that alcohol SBI is not being fully implemented as recommended. If alcohol SBI is implemented as recommended by USPSTF $(3,5,7)$ and coupled with population-level interventions recommended by the U.S. Community Preventive Services Task Force for the prevention of excessive drinking (e.g., increasing alcohol taxes and regulating alcohol outlet density $\mathbf{9 9}$ ), an opportunity exists to also reduce alcohol-related morbidity and mortality. Working to implement alcohol SBI at a systems level, including the provision of the new HEDIS measure, Unhealthy Alcohol Use Screening and Follow-Up, can improve alcohol SBI's use and benefit in primary care.

\footnotetext{
99 https://www.cdc.gov/ncbddd/fasd/alcohol-screening.html.

*** https://www.cdc.gov/ncbddd/fasd/documents/alcoholsbiimplementationguide.pdf.

t†† https://health.gov/dietaryguidelines/2015/.

$\$ \$ \$$ https://www.cdc.gov/alcohol/fact-sheets/binge-drinking.htm.

999 https://www.thecommunityguide.org/topic/excessive-alcohol-consumption.
}

\section{Summary}

What is already known about this topic?

Binge drinking increases the risk for adverse health conditions and death. Alcohol screening and brief intervention (SBI), recommended by the U.S. Preventive Services Task Force (USPSTF) for all adults in primary care, is effective in reducing binge drinking.

What is added by this report?

In $2017,81 \%$ of survey respondents were asked by their health care provider about alcohol consumption and $38 \%$ about binge drinking at a checkup in the past 2 years. Among those asked about alcohol use and who reported current binge drinking, $80 \%$ received no advice to reduce their drinking.

What are the implications for public health practice?

Implementation of alcohol SBI as recommended by USPSTF, coupled with population-level evidence-based interventions, can reduce binge drinking among U.S. adults.

\section{Acknowledgments}

Behavioral Risk Factor Surveillance System state coordinators from the states of Alabama, Alaska, Arizona, Arkansas, California, Colorado, Connecticut, Kansas, Nebraska, Nevada, New Hampshire, Tennessee, Wisconsin, and the District of Columbia; William Garvin, Machell Town, National Center for Chronic Disease Prevention and Health Promotion, CDC; Pat Santora, Substance Abuse and Mental Health Services Administration; Doug Kanovsky, Junqing Liu, Fern McCree, Sarah H. Scholle, National Committee for Quality Assurance.

Corresponding author: Lela R. McKnight-Eily, LMcKnightEily@cdc.gov, 404-498-2401.

\footnotetext{
${ }^{1}$ Division of Birth Defects and Infant Disorders, National Center on Birth Defects and Developmental Disabilities, CDC; ${ }^{2}$ Division of Human Development and Disability, National Center on Birth Defects and Developmental Disabilities, CDC; ${ }^{3}$ Office of the Director, National Center on Emerging Zoonotic and Infectious Diseases, CDC.
}

All authors have completed and submitted the International Committee of Medical Journal Editors form for disclosure of potential conflicts of interest. No potential conflicts of interest were disclosed.

\section{References}

1. Stahre M, Roeber J, Kanny D, Brewer RD, Zhang X. Contribution of excessive alcohol consumption to deaths and years of potential life lost in the United States. Prev Chronic Dis 2014;11:E109. https://doi. org/10.5888/pcd11.130293

2. World Health Organization. Global status report on alcohol and health 2018. Geneva, Switzerland: World Health Organization; 2018. https:// www.who.int/substance_abuse/publications/global_alcohol_report/en/

3. Curry SJ, Krist AH, Owens DK, et al.; US Preventive Services Task Force. Screening and behavioral counseling interventions to reduce unhealthy alcohol use in adolescents and adults: US Preventive Services Task Force recommendation statement. JAMA 2018;320:1899-909. https://doi. org/10.1001/jama.2018.16789

4. Bazzi A, Saitz R. Screening for unhealthy alcohol use. JAMA 2018;320:1869-71. https://doi.org/10.1001/jama.2018.16069 
5. O'Connor EA, Perdue LA, Senger CA, et al. Screening and behavioral counseling interventions to reduce unhealthy alcohol use in adolescents and adults: updated evidence report and systematic review for the US Preventive Services Task Force. JAMA 2018;320:1910-28. https://doi. org/10.1001/jama.2018.12086

6. McKnight-Eily LR, Okoro CA, Mejia R, et al. Screening for excessive alcohol use and brief counseling of adults -17 states and the District of Columbia, 2014. MMWR Morb Mortal Wkly Rep 2017;66:313-9. https://doi.org/10.15585/mmwr.mm6612a1

7. Jonas DE, Garbutt JC, Brown JM, et al. Screening, behavioral counseling, and referral in primary care to reduce alcohol misuse. AHRQ report no. 12-EHC055-EF. Rockville, MD: Agency for Healthcare Research and Quality; 2012. https://pubmed.ncbi.nlm.nih.gov/22876371screening-behavioral-counseling-and-referral-in-primary-care-to-reducealcohol-misuse-internet/
8. Froehlich-Grobe K, Jones D, Businelle MS, Kendzor DE, Balasubramanian BA. Impact of disability and chronic conditions on health. Disabil Health J 2016;9:600-8. https://doi.org/10.1016/j.dhjo.2016.04.007

9. Goplerud E, McPherson TL. Implementation barriers to and facilitators of screening, brief intervention, referral, and treatment (SBIRT) in federally qualified health centers (FQHCs). Chicago, IL: NORC at the University of Chicago, 2015. https://aspe.hhs.gov/report/ implementation-barriers-and-facilitators-screening-brief-interventionreferral-and-treatment-sbirt-federally-qualified-health-centers-fqhcs

10. National Institutes of Health; National Institute on Alcohol Abuse and Alcoholism. Helping patients who drink too much: a clinician's guide. Bethesda, MD: US Department of Health and Human Services; 2005. https://www.integration.samhsa.gov/clinical-practice/Helping_Patients_ Who_Drink_Too_Much.pdf 